\title{
Optimization of BEM-based Cooling Channels Injection Moulding Using Model Reduction
}

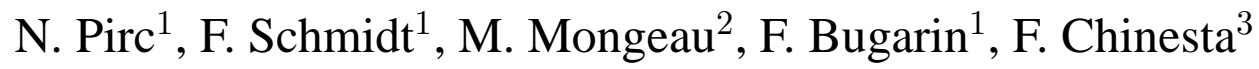

\author{
${ }^{1}$ CROMeP - Ecole des Mines d'Albi, Campus Jarlard,8 1013 Albi, cédex 9, France. \\ URL: www.enstimac.fr/recherche/cromep \\ e-mail: nicolas.pirc@enstimac.fr; \\ ${ }^{2}$ Institut de Mathématiques, Université de Toulouse, UPS, 31062 Toulouse cédex 9, France. \\ URL: www.mip.ups-tlse.fr/ \\ ${ }^{3}$ ENSAM-Paris, 151 boulevard de l'hôpital, 75013 Paris, France. \\ URL: www.paris.ensam.fr/
}

\begin{abstract}
Today, around 30\% of manufactured plastic goods rely on injection moulding. The cooling time can represent more than $70 \%$ of the injection cycle. In this process, heat transfer during the cooling step has a great influence both on the quality of the final parts that are produced, and on the moulding cycle time. Models based on a full 3D finite element method renders unpractical the use of optimization of the design and placement of the cooling channel in injection moulds. We have extended the use of boundary element method (BEM) to this process. We introduce in this paper a practical methodology to optimize both the position and the shape of the cooling channels in injection moulding processes. We couple the direct computation with an optimization algorithm such as SQP (Sequential Quadratic Programming). First, we propose an implementation of the model reduction in the BEM solver. This technique permits to reduce considerably the computing time during the linear system resolution (unsteady case). Secondly, we couple it with an optimization algorithm to evaluate its potentiality. For example, we can minimize the maximal temperature on the cavity surface subject to a temperature uniformity constraint. Thirdly, we present encouraging computational results on plastic parts that show that our optimization methodology is viable.
\end{abstract}

KEYWORDS: BEM, optimization, reduction model, injection moulding, SQP.

\section{INTRODUCTION}

Numerical simulations for designing injection moulds have become an important developement in injection moulding processes. The location of the cooling channels is a major element in the design of the mould because the cooling time can represents up to $70 \%$ of the injection cycle. We need efficient numerical simulations in order to optimize the process parameters, but models based on full 3D finite element method renders unpractical the use of optimization for this design and placement of cooling channels in injection moulds.

In this context, the Dual Reciprocity Method (DRM), introduced by Brebbia [1], is acknowledged to be one of the most effective BEM techniques for transforming domain integrals into boundary integrals. More- over, during the optimization, this method permits to compute exact gradients, thereby avoiding the $\mathrm{N}$ direct computations per optimization iteration that are needed by finite-difference gradient approximation (where $\mathrm{N}$ is the number of optimization variables). However, thermal models involved in the numerical modelling in injection moulding processes a certain number of numerical difficulties such as size meshing, long simulations, or the necessity to define a homogenized thermal conductivity. In the first part of this paper, we present the use of boundary element method (BEM) and DRM applied to unsteady heat transfer of injection moulds. The BEM software, developed at the CROMeP laboratory [2], was combined with an adaptive reduced modelling [3]. This procedure permits to reduce considerably the computing time during the linear system resolution in unsteady prob- 
lem. Then, we present a practical methodology to optimize both the position and the shape of the cooling channels in injection moulding processes. We couple the direct computation with an optimization algorithm such as SQP (Sequential Quadratic Programming) [4].

For the sake of simplicity, we will consider a potential problem defined in a $2 \mathrm{D}$ unbounded domain. The capabilities of both the reduced order modeling and the boundary element method will be outlined.

\section{BEM AND DRM APPLIED TO POISSON EQUATION}

Using BEM, only the boundary of the domain has to be meshed and internal points are explicitly excluded from the solution procedure. An interesting side effect is the considerable reduction in size of the linear system to be solved [5]. The transient heat conduction in a homogeneous isotropic body $\Omega$ is described by the diffusion equation [6], where $a$ is the material diffusion:

$$
\forall M \in \Omega, \quad \vec{\nabla}^{2} T(M, t)=\frac{1}{a} \frac{\partial(M, t)}{\partial t}
$$

We define the initial conditions and the boundary conditions as:

$$
\left\{\begin{array}{l}
T(M, t)=T_{0} \forall M \in \Gamma_{P} \\
\phi(M, t)=\lambda .\left(T-T_{C}\right) \forall M \in \Gamma_{C} \\
T(M, t=0)=T^{0}(M)
\end{array}\right.
$$

Where $\Gamma_{P}$ is the boundary of the polymer and $\Gamma_{C}$ the boundary of the channels. The temperature of the coolant is $T_{C}$ and the heat transfer coefficient, $h$, is related to the coolant flow rate (via Colburn correlation). Several strategies are possible to solve such problems using BEM. Mätzig [7] propose to use space and time Green's function. To express the domain integral in terms of equivalent boundary integrals, we introduce the DRM approximation [1]. The solution is defined as a series of particular solutions $\hat{T}_{k}$ located in each boundary nodes $N_{n}$, and each internal nodes $N_{i}$. We obtain Eq (3), explain in detail by Mathey [2]

$$
\begin{aligned}
& C_{i} T_{i}-a \int_{\Gamma} T \cdot q^{*} d \Gamma-\int_{\Gamma} q \cdot T^{*} d \Gamma \\
= & \sum_{k=1}^{N_{n}+N_{i}} \beta_{k}\left(C_{i} \hat{T_{i k}}+\int_{\Gamma} \hat{T_{k}} \cdot q^{*} d \Gamma-\int_{\Gamma} \hat{q_{k}} \cdot T^{*} d \Gamma\right)
\end{aligned}
$$

Here, $T$ and $q$ denotes the temperature and the flux denotes, and $C_{i}$ is equal to 1 since the point $i$ is inside the domain and to 0.5 on its regular boundary. The following Green's function $T^{*}$ and $q^{*}$ [1] denotes the fundamental solution of this equation. The vector $\beta$ is define such as:

$$
\underline{\beta}=\frac{1}{a} \underline{F}^{-1} \underline{\dot{T}}
$$

Matrix $\underline{\underline{F}}$ consists of interpolation-function values $f=1+r$ at each point.

\section{REDUCED MODELING}

Usual reduced models perform the simulation of some similar problem or the desired one in a short time interval. From these solutions, the KarhunenLoève decomposition [3] can be performed, allowing to extract the most relevant functions describing the solution evolution.

\subsection{The Karhunen-Loève decomposition}

We assume that the evolution of a certain field $T(x, t)$ is known. In practical applications, this field is defined at the spatial mesh nodes $x_{i}$ (with $i \in\{1, \cdots, N\}$ ), and for some time $t^{m}=$ $m . \Delta t$ with $m \in\{1, \cdots, M\}$. We introduce the notation $T^{m}\left(x_{i}\right)$ for defining the vector containing the nodal degrees of freedom (temperatures) at time $t^{m}$. The main idea of the Karhunen-Loève (KL) decomposition tell us how to obtain the most typical or characteristic structure $\phi(x)$ among these $T^{m}(x) \forall M$. This is equivalent to obtaining a function $\phi(x)$ maximizing $\alpha$ defined as:

$$
\alpha=\frac{\sum_{p=1}^{P}\left[\sum_{i=1}^{N} \phi\left(x_{i}\right) T^{p}\left(x_{i}\right)\right]^{2}}{\sum_{i=1}^{N}\left(\phi\left(x_{i}\right)\right)^{2}}
$$

This leads to:

$$
\begin{array}{r}
\sum_{p=1}^{P}\left[\left[\sum_{i=1}^{N} \widetilde{\phi}\left(x_{i}\right) T^{P}\left(x_{i}\right)\right]\left[\sum_{i=1}^{N} \phi\left(x_{i}\right) T^{P}\left(x_{i}\right)\right]\right] \\
=\alpha \sum_{i=1}^{N} \widetilde{\phi}\left(x_{i}\right) \phi^{P}\left(x_{i}\right) \quad \forall \widetilde{\phi}
\end{array}
$$

where $\widetilde{\phi}$ denotes the variation of $\phi(x)$ which can be rewritten under the form:

$$
\underline{\tilde{\phi}}^{T} \underline{\underline{k}} \cdot \underline{\Phi}=\alpha \underline{\tilde{\phi}}^{T} \cdot \underline{\phi} \forall \underline{\tilde{\phi}}^{T} \Rightarrow \underline{\underline{k}} \cdot \underline{\phi}=\alpha \cdot \underline{\phi}
$$


We define the matrix $\mathrm{Q}$ containing the discrete field history, and the vector $\phi$ such that its i-component is $\phi\left(x_{i}\right)$. This yields to the eigenvalue problem $\underline{\underline{D}}=$ $\underline{\underline{Q}} \underline{\underline{Q}}^{T}$ :

$$
Q=\left(\begin{array}{cccc}
T_{1}^{1} & T_{2}^{1} & \cdots & T_{P}^{1} \\
T_{1}^{2} & T_{2}^{2} & \cdots & T_{1}^{P} \\
\vdots & \vdots & \ddots & \vdots \\
T_{N}^{1} & T_{N}^{2} & \cdots & T_{N}^{P}
\end{array}\right)
$$

The functions defining the most characteristic structure of $T^{P}(x)$ are the eigenfunctions $\phi_{n}(x) \equiv \phi_{n}$ associated with the largest eigenvalues.

\subsection{A posteriori reduced model}

We solve the eigenvalue problem defined by Eq (7) selecting the eigenfunctions $\phi_{n}$ associated with the eigenvalues belonging to the interval defined by the largest eigenvalue such as $\Phi_{n}$ 's sum is upper or equal to $99.9 \%$ of $\Phi_{N}$ 's sum. In practice, $n$ is much lower than $N$. Let us now try to use these $\mathrm{n}$ eigenfunctions $\phi_{n}$ for approximating the solution. Let $B$ be the following matrix:

$$
\underline{\underline{B}}=\left(\begin{array}{cccc}
\phi_{1}\left(X_{1}\right) & \phi_{2}\left(X_{1}\right) & \cdots & \phi_{n}\left(X_{1}\right) \\
\phi_{1}\left(X_{2}\right) & \phi_{2}\left(X_{2}\right) & \cdots & \phi_{n}\left(X_{2}\right) \\
\vdots & \vdots & \ddots & \vdots \\
\phi_{1}\left(X_{N}\right) & \phi_{2}\left(X_{N}\right) & \cdots & \phi_{n}\left(X_{N}\right)
\end{array}\right)
$$

We express the linear system of equations resulting from the semi-implicit thermal-model discretization as:

$$
\underline{T}^{m+1}=\sum_{i=1}^{i=n} \zeta_{i}^{m+1} \phi_{i}=\underline{\underline{B}} \cdot \underline{\zeta}^{m+1}
$$

\section{MOULD COOLING OPTIMIZATION}

Each optimization iteration involves performing a BEM simulation and computing the objective and constraint functions. The optimization method allows updating the cooling channel design parameters (subject to the constraints) until a minimum of the cost function is reached [5]. Figure 1 shows the coupling between the thermal solver and the optimization algorithm.

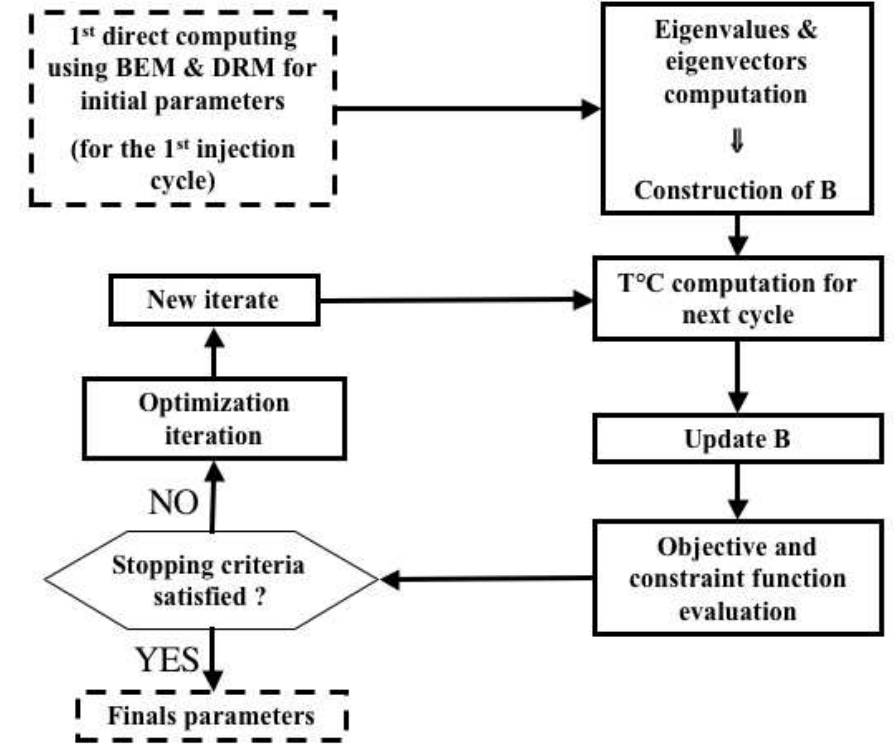

Figure 1: optimization procedure

The SQP method is designed for monoobjective optimization [4]. However, pratical optimization problems almost always involves at least two objective functions. One way to proceed in such a context is to consider as cost function a weighted sum of two objectives, but this method involves choosing a weighting parameter. We rather propose here using one objective as optimization criterion, and the other as a non-linear constraint. The first criterion involves miniming the maximal temperature on the cavity surface. The second criterion aims at improving temperature uniformity. More precisely, we formulate our problem under the form:

$$
\begin{gathered}
\operatorname{minimize} \max _{i \in D}\left(T_{i}\right) \\
\text { subject to } \sum_{i \in D}\left|T_{i}-T_{\text {moy }}\right| \leq \sigma
\end{gathered}
$$

where $\mathrm{D}$ is the set of discretization elements of the plastic part where the temperature $\mathrm{T}_{i}$ is measured, $\mathrm{T}_{\text {moy }}$ is the average of the $\mathrm{T}_{i}$ 's, and $\sigma$ is a user-defined temperature uniformity tolerance, fixed here equal to 4.

\section{APPLICATION TO TWO-DIMENSIONAL CASE}

It is important to note that using reduced model to optimize the cooling channel location is possible since the vector $\phi_{n}\left(x_{N}\right)$ does not change when the 
unsteady equation source term changes, i.e. even if the position of the cooling channels changed.

Optimization variables are the coordinates $\left(X_{i}, Y_{i}\right)$ of each circle center $i$, and the radius of them. This geometry have 7 channels, thus we have 21 optimization variables in our problem. The coolant temperature is fixed as $T_{C}=30^{\circ} \mathrm{C}$. Figure 2 displays the geometry used to validate our method. Dotted circles show the initial configuration of the cooling channels, and bold circles show the optimized position.

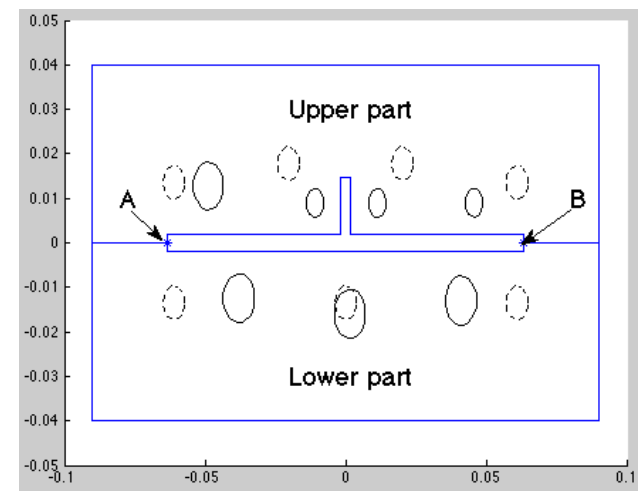

Figure 2: Channels configuration before and after optimization

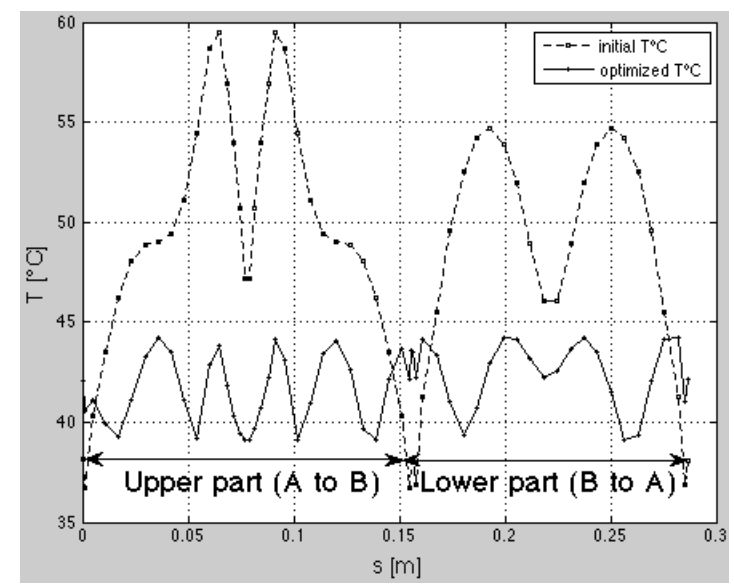

Figure 3: Temperature before and after optimization

On average, one objective function evaluation requires 8 seconds of CPU time on a Macintosh 1.83 GHz Intel Core 2 Duo, and 22 iterations and 534 evaluations are necessary to reach convergence.

Table 1: CPU time comparison

\begin{tabular}{lll}
\hline Method & direct computation & optimization \\
\hline DRM & 38.2 seconds & 5.6 hours \\
reduction model & 1 second & 9.6 minutes \\
\hline
\end{tabular}

The reduced model permits to divide by more than 40 the CPU of each direct computation, compared to DRM. We used $n=17$ in our simulations, whereas $N=288$ (nodes number).

\section{CONCLUSIONS}

Our methodology uses BEM to solve the unsteady heat transfer equation during the cooling step of the injection moulding process. Simulation results are used in an optimization procedure to find the best geometry and process parameters according to a given objective function. Reduce model technique involves a Karhunen-Love decomposition leading to an optimal number of approximation functions, allowing to considerable CPU time savings (some times in the order of 40). Our preliminary test showed that our approach is viable for optimizing the design of cooling channels for injection moulding. Various objective functions can be provided by the user (either directly as a cost function or within constraints). We presently work on more complex 3D moulds with more general parameterizations of the cooling channels.

\section{REFERENCES}

[1] C. S. Chen, C. A. Brebbia, H. Power. Dual reciprocity method using compactly supported radial basis functions Communications in Numerical Methods in Engineering, editors John Wiley \& Sons, Vol 15, 2, pp. 137-150, 1999.

[2] E Mathey, L Penazzi, FM Schmidt, F Rond-Oustau. Automatic optimization of the cooling of injection mold based on the boundary element method Materials Processing and Design: Modeling, Simulation and Applications, Proc. NUMIFORM"04, Vol 712, pp. 222-227, 2004.

[3] F. Chinesta, A. Ammar, F. Lemarchand, P. Beauchchene, F. Boust. Alleviating mesh constraints: model reduction, parallel time integration and high resolution homogenization. Comput. Methods Appl. Mech. Engrg, editors Elsevier, 2007.

[4] J. Nocedal and S. S. J. Wright Numerical optimization series in operation research, editors springer, 1999.

[5] N. Pirc, F. Schmidt, M. Mongeau, F. Bugarin,. BEM-based cooling optimization for 3D injection molding. International Journal of Mechanical Sciences , Proc. ASMDO'07, Vol 48, 4, pp. 430-439, 2006.

[6] S. Kenig, M.R. Kamal. Cooling molded parts, a rigorous analysis. Soc. Plast. Eng. J., Vol 26, pp. 5057, 1970.

[7] B. A. Davis,P.J. Gramann, J.C. Mätzig,T.A. Osswald. The dual reciprocity method for heat transfer in polymer processing. Eng. anal. bound. elem., editor, Elsevier, vol. 13, 3, pp. 249-261, 1994. 